\title{
Utilização de conchas de Anomalocardia brasiliana como substrato de biofiltros no tratamento dos efluentes de cultivo heterotrófico de camarão marinho em laboratório
}

\author{
Clamshell (Anomalocardia brasiliana) use as biofilter \\ substrate in the treatment of heterotrophic shrimp \\ culture in laboratory
}

Laboratốrio de Maricultura Sustentāvel (LAMARSU), Departamento de Pesca e Aquicultura, Universidade Federal Rural de Pernambuco (UFRPE) Av. Dom Manoel de Medeiros, s/n, Dois Irmãos, CEP 52171-900, Recife, PE, Brasil

${ }^{2}$ Escritôrio Regional da llha de Itamaracá, Instituto Agronômico de Pernambuco (IPA),

lha de Itamaracă, PE, Brasil

*autor correspondente

凶srsnmt@hotmail.com
RESUMO: Este trabalho teve por finalidade comparar a eficiência de biofiltros de concha de marisco e anéis de cerâmica no tratamento de efluentes. $\mathrm{O}$ experimento foi realizado na Universidade Federal Rural de Pernambuco - UFRPE, no Laboratório de Maricultura Sustentável - LAMARSU. O delineamento inteiramente casualizado considerou três tratamentos, com três repetições cada, em um total de nove unidades experimentais, divididos em: substrato natural, com conchas de mariscos (SNCM), substrato comercial com anéis de cerâmica (SCAC) e controle. A comparação dos tratamentos foi feita através da ANOVA e o teste de Duncan $(\mathrm{P}<0,05)$. Os resultados demonstram que os tratamentos SNCM e SCAC alcançaram níveis de redução de amônia diferentes apenas no primeiro dia e iguais no restante do período experimental, chegando a uma redução de zero. Estes dados foram melhores do que no controle, no qual houve uma redução da amônia de $0,60 \mathrm{mg} / \mathrm{L}$ a $0,39 \mathrm{mg} / \mathrm{L}$. Desta maneira, conclui-se que o substrato de concha de marisco é um material que pode substituir ou atuar em conjunto ao anel de cerâmica, objetivando a redução de amônia do meio.

PALAVRAS-CHAVE: Substrato natural, filtro biológico, amônia, bivalve.
ABSTRACT: The purpose of this research was to compare the efficiency of clamshell and ceramic ring biofilters in the treatment of aquaculture effluents. The experiment was carried out in the Laboratory of Mariculture Development - LAMARSU at the Federal Rural University of Pernambuco - UFRPE. The completely randomized design considered three treatments with three replicates each, in a total of nine experimental units, divided in natural clamshell substrates (NCS), ceramic ring commercial substrates (CRCS), and without any type of substrate (control). The comparison between treatments was made using ANOVA and Duncan test $(P<0.05)$. Results showed that the NCS and CRCS treatments presented different levels of ammonia reduction only on the first day; ammonia levels were the same for both treatments in the remainder of the trial period, coming to a reduction to zero. These figures were better than those found in the control treatment, where there was a reduction of ammonia from $0.60 \mathrm{mg} / \mathrm{L}$ to $0.39 \mathrm{mg} / \mathrm{L}$. Thus, it was possible to conclude that the natural clamshell substrate (NCS) can replace or be used in conjunction with the ceramic ring commercial substrate (CRCS) aiming at ammonia reduction in the medium.

KEYWORDS: Natural substrate, biological filter, ammonia, clam. 


\section{Introdução}

A conservação da água tem sido um dos principais pontos de estudo na aquicultura nos últimos anos. $\mathrm{O}$ avanço no uso de técnicas de controle da qualidade de água em escala comercial na produção de peixes iniciou-se a partir da última década (MASSER; RAKOCY; LOSORDO, 1999).

O tipo de sistema de cultivo adotado em fazendas de camarão influencia no volume e na quantidade dos efluentes produzidos, tendo relação com o manejo e layout da fazenda. Segundo Avnimelech (2009), em média 25\% dos nutrientes adicionados como alimento são incorporados pelos animais, o restante permanece no ambiente, podendo causar eutrofização.

Para o tratamento de efluentes, têm sido propostos métodos físicos e biológicos. Entre os métodos físicos, destacam-se aqueles que propõem o uso de tanques de sedimentação. Os métodos biológicos, por sua vez, consideram que os efluentes, por serem ricos em nutrientes e micro-organismos, tornam-se potencialmente apropriados para o cultivo de moluscos bivalves e macroalgas (MODESTO et al., 2010).

A técnica de reciclagem de efluentes, através de biofiltros, consiste em incorporar tecnologia para recuperar a qualidade da água, uma vez que essa já tenha passado por todas as unidades de cultivo. Inicialmente, ocorre a remoção de sólidos suspensos por filtros mecânicos e, logo após, o efluente passa por um biofiltro, para que ocorra a conversão da amônia em nitrito e nitrato realizada por bactérias quimioautotróficas (GUIMARÃES, 2005).

O período de maturação que é o tempo necessário para colonização das bactérias quimioautotróficas e o monitoramento da amônia são ferramentas para avaliar a eficiência dos bioflitros. (MASSER; RAKOCY; LOSORDO, 1999; GUTIERREZ-WING; MALONER, 2006).

Neste contexto, esta pesquisa teve como objetivo comparar a eficiência de um substrato natural de conchas de marisco Anomalocardia brasiliana com um substrato comercial de anéis de cerâmica, no tratamento de efluentes do cultivo de Litopenaeus vannamei em meio heterotrófico.

\section{Material e Métodos}

\section{Desenho experimental}

O experimento foi realizado no Laboratório de Maricultura Sustentável - LAMARSU, do Departamento de Pesca e Aquicultura - DEPAQ da Universidade Federal Rural de Pernambuco-UFRPE. Os sistemas de substratos testados foram divididos em três tratamentos, com três repetições cada, totalizando nove unidades experimentais em delineamento inteiramente casualizado.

Os tratamentos foram divididos em: substrato natural com conchas de mariscos de Anomalocardia Brasiliana (SNCM); substrato comercial com anéis de cerâmica (SCAC); nenhum tipo de substrato (controle).

\section{Preparação e maturação dos Biofiltros}

Os substratos de conchas de Anomalocardia brasiliana e anéis de cerâmica foram higienizados com água doce e expostos ao sol por um período de 24 horas. Depois deste período, foram lavados e colocados dentro dos compartimentos dos biofiltros externos de aquário modelo "JEBO external 838" e, posteriormente, na caixa de maturação com volume de $500 \mathrm{~L}$.

Para uma colonização uniforme dos substratos, foi utilizada uma fonte de água com uma concentração de 3,0 mg/L de amônia. Depois do processo de maturação dos biofiltros, foram separados em suas respectivas unidades experimentais.

\section{Efluentes do meio heterotrófico}

Os efluentes utilizados neste experimento foram provenientes de um cultivo de Litopenaeus vannamei em meio heterotrófico. Inicialmente, o efluente foi armazenado em uma caixa d'água com um volume de $500 \mathrm{~L}$, por um período de três dias. Antes de iniciar o experimento, a água foi submetida a uma pré-filtragem através de uma bateria de filtros mecânicos tipo cartucho (5, $3 \mu \mathrm{m}$ e $1 \mu \mathrm{m}$ ) para a retirada de todo material em suspensão, sendo, posteriormente, distribuída às unidades experimentais. Cada tratamento contou com um volume de $60 \mathrm{~L}$ de água, a ser tratada durante 4 dias. As características da água dos efluentes foram: temperatura média de $28^{\circ} \mathrm{C}$, salinidade de 36 , oxigênio dissolvido de 3,6 mg/L e concentração de nitrogênio total de $0,81 \mathrm{mg} / \mathrm{L}$.

\section{Qualidade de água}

Diariamente, foram mensuradas as seguintes variáveis físico-químicas da água: temperatura e oxigênio dissolvido (oxímetro digital YSI modelo 5512 FT); salinidade (refratômetro modelo S-10, marca ATAGO); pH (medidor digital, modelo L 55, marca HANNA) e amônia total pelo método de Koroleff (1976).

\section{Análise estatîstica}

Os resultados foram submetidos ao teste de Shapiro-Wilk, para verificar a normalidade dos dados e, posteriormente, analisados através de ANOVA e teste de Duncan $(\mathrm{P}<0,05)$. Para análise, foi utilizado o programa STATISTICA versão 8.

\section{Resultados e Discussão}

A colonização das bactérias foi observada ao oitavo dia, ocasionando uma redução da concentração da amônia total para $0,5 \mathrm{mg} / \mathrm{L}$ (Figura 1 ).

Durante o experimento, no tratamento controle, as concentrações médias foram temperatura de $31^{\circ} \mathrm{C} \pm 0,35^{\circ} \mathrm{C}$, salinidade de $35,1 \pm 1,1$, oxigênio dissolvido de $3,8 \mathrm{mg} / \mathrm{L} \pm 0,3 \mathrm{mg} / \mathrm{L}$ e pH 8,19 $\pm 0,09$. No tratamento SNCM, foram usados temperatura de $30,5{ }^{\circ} \mathrm{C} \pm 0,68{ }^{\circ} \mathrm{C}$, salinidade de 35,9 \pm 0,95, oxigênio dissolvido de 3,9 mg/L $\pm 0,3 \mathrm{mg} / \mathrm{L}$

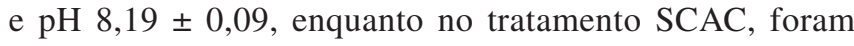
utilizados temperatura de $30,9{ }^{\circ} \mathrm{C} \pm 0,32{ }^{\circ} \mathrm{C}$, salinidade de $35,8 \pm 1,1$, oxigênio dissolvido de 4,0 mg/L $\pm 0,1 \mathrm{mg} / \mathrm{L} \mathrm{e}$ pH $8,17 \pm 0,09$. Não houve diferença significativa entre os tratamentos $(\mathrm{P}<0,05)$ para estas variáveis, durante o período experimental (Tabela 1).

No primeiro dia do experimento, foi observada uma redução na concentração $(\mathrm{mg} / \mathrm{L})$ da amônia total de 0,81 para 
Tabela 1. Média e desvio padrão das variáveis da água: temperatura, salinidade, oxigênio dissolvido e pH nos tratamentos, durante os quatro dias de experimento.

\begin{tabular}{ccccc}
\hline Tratamentos & Temperatura $\left({ }^{\circ} \mathbf{C}\right)$ & Salinidade & Oxigênio dissolvido (mg.L) & pH \\
\hline SNCM & $30,5 \pm 0,68$ a & $35,9 \pm 0,95$ a & $3,9 \pm 0,3 \mathrm{a}$ & $8,19 \pm 0,09$ a \\
SCAC & $30,9 \pm 0,32 \mathrm{a}$ & $35,8 \pm 1,10 \mathrm{a}$ & $4,0 \pm 0,1 \mathrm{a}$ & $8,17 \pm 0,09 \mathrm{a}$ \\
Controle & $31,0 \pm 0,35 \mathrm{a}$ & $35,1 \pm 1,10 \mathrm{a}$ & $3,8 \pm 0,3 \mathrm{a}$ & $8,19 \pm 0,09 \mathrm{a}$ \\
\hline
\end{tabular}

Médias seguidas da mesma letra, por coluna, não diferiram significativamente pelo teste de Ducan $(\mathrm{P}<0,05)$.

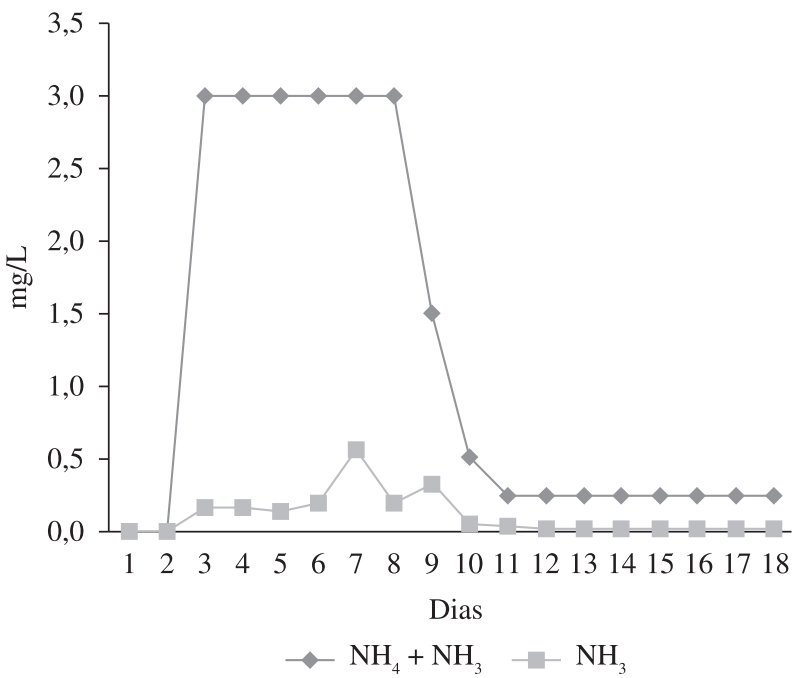

Figura 1. Concentração $(\mathrm{mg} / \mathrm{L})$ de amônia total $\left(\mathrm{NH}_{4}+\mathrm{NH}_{3}\right)$ durante o período de maturação dos biofiltros.

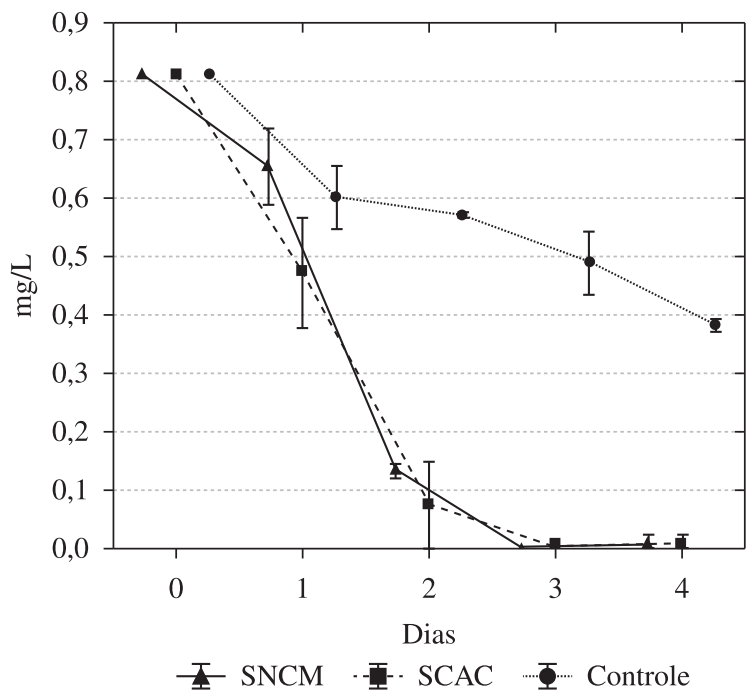

Figura 2. Concentração de amônia total nos tratamentos SNCM, SCAC e controle durante o período experimental.

$0,60 \pm 0,02$ no tratamento controle, de 0,81 para $0,65 \pm 0,02$ no tratamento SNCM e de 0,81 para $0,47 \pm 0,04$, no tratamento SCAC (Figura 2).

A amônia é o principal produto de excreção dos organismos aquáticos, resultado do catabolismo das proteínas, principalmente da degradação da matéria orgânica realizada por bactérias. A oxidação deste composto ocorre por meio de bactérias quimioautotróficas dos gêneros Nitrosomonas e
Nitrobacter (ALVES; MELLO, 2007; QUEIROZ; BOEIRA, 2007).

Segundo Boyd e Tucker (1992), os limites do nitrogênio total em viveiros de aquicultura devem estar entre $2,0 \mathrm{mg} / \mathrm{L} \mathrm{e}$ 3,0 mg/L, sendo que, para a maioria dos peixes e crustáceos de águas quentes, a concentração letal (breve exposição de 24 horas a 96 horas) de amônia não ionizada é de $0,4 \mathrm{mg} / \mathrm{L}$ a 2,0 mg/L (QUEIROZ; BOEIRA, 2007). Segundo Kubitza e Cyrino (1999), concentrações de amônia total acima 0,20 mg/L já são consideradas letais para diversos organismos aquáticos.

No decorrer do experimento, foram observadas reduções da concentração de amônia total na água para os três tratamentos. Ao final do experimento, foi observado que, nos tratamentos SNCM e SCAC, houve uma redução para zero, proporcionando uma redução na concentração de amônia total significativamente mais eficiente do que no controle que reduziu de $0,60 \mathrm{mg} / \mathrm{L}$ para $0,39 \mathrm{mg} / \mathrm{L}$ (Figura 2).

Os valores de redução de amônia observados no presente trabalho, tanto no SNCM como para o SCAC, assemelham-se ao encontrado por Davidson et al. (2008), que, utilizando diferentes biofiltros de areia, obteve redução de $0,81 \mathrm{mg} / \mathrm{L}$ para 0,11 mg/L; Lobão (1998), que, utilizando biofiltros com substrato de areia na larvicultura de Macrobrachium rosenbergii, manteve os níveis abaixo de 1,0 mg/L; Singh (1999), ao utilizar biofiltros com meio plástico e também a concentração abaixo de 0,20 mg/L, e Tseng (1998), ao utilizar biofiltros com meio plástico durante o cultivo experimental de $P$. monodon, obtiveram uma redução de 3,64 mg/L para 0,20 mg/L, após um período de cerca de 6 dias.

\section{Conclusões}

O substrato natural de concha de marisco obteve níveis de redução de amônia com a mesma eficiência que o anel de cerâmica, sendo de grande importância com relação à economia de material que compõe um biofiltro.

\section{Referências}

ALVES, C. S.; MELLO, G. L. Manual prático de monitoramento de qualidade de água e solo em aquicultura. Recife: FAEPE, 2007. 47 p.

AVNIMELECH, Y. Biofloc technology: a pratical guide book. Baton Rouge: The World Aquaculture Society, 2009. 182 p.

BOYD, C. E.; TUCKER, C. S. Water quality and pond soil analyses for aquaculture. Auburn: Auburn University - Alabama Agricultural Experiment Station, 1992. 183 p. 
DAVIDSON, J.; HELWIG, N.; SUMMERFELT, S. T. Fluidized sand biofilters used to remove ammonia, biochemical oxygen demand, total coliform bacteria, and suspended solids from an intensive aquaculture effluent. Aquacultural Engineering, v. 39, p. 6-15, 2008. http://dx.doi.org/10.1016/j.aquaeng.2008.04.002

GUIMARÃES, I. M. Dimensionamento de um sistema de recirculação de água para um laboratório de larvicultura de moluscos. 2005. 40 f. Monografia de conclusão de curso (Graduação em Engenharia de Pesca)-Universidade Federal Rural de Pernambuco, Departamento de Pesca e Aquicultura, Recife, 2005.

GUTIERREZ-WING, M. T.; MALONER, F. Biological filters in aquaculture: trends and research directions for freshwater and marine applications, Aquacultural Engineering, v. 34, p. 163-171, 2006. http://dx.doi.org/10.1016/j.aquaeng.2005.08.003

KOROLEFF, F. Determination of nutrients. In: GRASSHOFF, K. Methods of seawater analysis. Verlag: Chemie Weinhein, 1976. p. 117-187.

KUBITZA, F.; CYRINO, J. E. P. Effects of feed quality and feeding practices on the quality of fish. In: CHANGY, K.; WANG, S. S. (Ed.). A Brazilian fish culture outlook. São Paulo: Technomic Publishing Company, 1999. p. 53-69.
LOBÃO, V. L. et al. Estudo comparativo entre quatro métodos de sistemas fechados de circulação em larvicultura Macrobrachium rosenbergii. Boletim do Instituto da Pesca, v. 25, p. 101-109, 1998.

MASSER, M. P.; RAKOCY, J.; LOSORDO, T. M. Recirculating aquaculture tank Production systems: management of recirculating systems. Stoneville: Southern Regional Aquaculture Center Publication, 1999. 452 p.

MODESTO, G. A. et al. Utilização de Crassotrea rhizophorae (Guilding, 1828) no tratamento dos efluentes do cultivo de Litopenaeus vannamei (Boone, 1931). Pan-American Journal of Aquatic Sciences, v. 5, p. 367-375, 2010.

QUEIROZ, J. F.; BOEIRA, R. C. Boas práticas de manejo (BPMs) para reduzir o acúmulo de amônia em viveiros de aquicultura. Jaguariúna: Embrapa Meio Ambiente, 2007. 5 p. (Embrapa Meio Ambiente. Comunico Técnico, n. 44).

SINGH, S.; EBELING, J.; WHEATON, F. Water quality trials in four recirculating aquacultural system configurations. Aquacultural Engineering, v. 20, p. 75-84, 1999. http://dx.doi.org/10.1016/ S0144-8609(99)00003-5

TSENG, K. F.; SU, H. M.; SU, M. S. Culture of penaeus monodon in a recirculating system. Aquacultural Engineering, v. 17 , p. $138-147,1998$. http://dx.doi.org/10.1016/S01448609(98)00011-9 